\title{
Nonparametric Single-Trial EEG Feature Extraction and Classification of Driver's Cognitive Responses
}

\author{
Chin-Teng Lin, ${ }^{1}$ Ken-Li Lin, ${ }^{1,2}$ Li-Wei Ko, ${ }^{1}$ Sheng-Fu Liang, ${ }^{3}$ Bor-Chen Kuo, ${ }^{4}$ and I-Fang Chung ${ }^{5}$ \\ ${ }^{1}$ Department of Electrical and Control Engineering and Brain Research Center, National Chiao-Tung University, \\ 1001 Ta Hsueh Road, Hsinchu 300, Taiwan \\ ${ }^{2}$ Computer Center of Chung Hua University, Hsinchu 707, Section 2, WuFu Road, HsinChu 300, Taiwan \\ ${ }^{3}$ Department of Computer Science and Information Engineering, National Cheng-Kung University, No. 1, University Road, \\ Tainan 701, Taiwan \\ ${ }^{4}$ Graduate Institute of Educational Measurement and Statistics, National Taichung University, 140 Min-Shen Road, \\ Taichung 40306, Taiwan \\ ${ }^{5}$ Institute of Biomedical Informatics, National Yang-Ming University, No. 155, Section 2, Linong Street, Taipei 112, Taiwan
}

Correspondence should be addressed to I-Fang Chung, ifchung@ym.edu.tw

Received 1 December 2007; Accepted 10 March 2008

Recommended by Chien-Cheng Lee

We proposed an electroencephalographic (EEG) signal analysis approach to investigate the driver's cognitive response to trafficlight experiments in a virtual-reality-(VR-) based simulated driving environment. EEG signals are digitally sampled and then transformed by three different feature extraction methods including nonparametric weighted feature extraction (NWFE), principal component analysis (PCA), and linear discriminant analysis (LDA), which were also used to reduce the feature dimension and project the measured EEG signals to a feature space spanned by their eigenvectors. After that, the mapped data could be classified with fewer features and their classification results were compared by utilizing two different classifiers including $k$ nearest neighbor classification (KNNC) and naive bayes classifier (NBC). Experimental data were collected from 6 subjects and the results show that NWFE+NBC gives the best classification accuracy ranging from $71 \% \sim 77 \%$, which is over $10 \% \sim 24 \%$ higher than LDA + KNN1. It also demonstrates the feasibility of detecting and analyzing single-trial EEG signals that represent operators' cognitive states and responses to task events.

Copyright (c) 2008 Chin-Teng Lin et al. This is an open access article distributed under the Creative Commons Attribution License, which permits unrestricted use, distribution, and reproduction in any medium, provided the original work is properly cited.

\section{INTRODUCTION}

Global urbanization, increases in urban populations, urban sprawl, the growth of "mega-cities," and the unrelenting increases in traffic around the world have created renewed concern about driving safety due to the growing number of traffic fatalities. Among these incidents, the fatalities are frequently caused by drivers' failure to perceive changes in traffic lights or unexpected conditions occurring on the roads. Preventing such accidents is thus a major focus of efforts in the field of active safety research and healthcare systems in vehicles. Early detection and recognition of driver's cognitive response could be helpful in giving precautious alarms or providing some preventive actions.

In recent studies [1-3], many researchers proposed to develop quantitative techniques for ongoing assessment of cognitive effort, engagement, and workload, by investigat- ing the neurobiological mechanisms underlying electroencephalographic (EEG) brain dynamics. A way to determine the relationship between different stimuli and human cognitive responses accompanying correct, incorrect, and absent motor responses is the use of event-related brain potential (ERP) signals. ERP signals related to many different stimuli such as audio, vision, pain, electric shock, emotion changes, and so forth can be observed with some latency (e.g., P300) as the stimulus event is given to or removed from a subject. Such important ERP features can be extracted and detected for the purpose of brain computer interface, for example, to choose characters or move a cursor on a computer screen [4-6]. Bayliss and Ballard $[7,8]$ designed an experiment to recognize the existence of P300 ERP epochs at red traffic lights and the absence of this signal at yellow traffic lights in a virtual driving environment. They have shown that building a brain computer interface using the P300 ERP would prove 
feasible. Based on Bayliss and Ballard's experimental design concept, we have also developed methods in our previous paper [9] for analyzing single-trial electrical recordings from the human scalp in traffic light experiments and have proved the efficacy. In this paper, we further simplified and refined the methodology for quantitative analysis for ongoing assessment of driver's cognitive responses by directly utilizing electroencephalographic (EEG) signals to investigate the neurobiological information underlying brain dynamics in traffic-light experiments in a virtual-reality (VR)-based simulated driving environment. Here, there is an important discrepancy between these two papers: the data collection process in this paper was modified by directly accessing brain signals originating in one channel of the visual region of the brain. Hence, we did not need to use blind source separation technique like independent component analysis (ICA) as adopted in our previous paper. This approach can eliminate the necessity of offline manual selection of desired source component such that online classification is possible and desirable in the sense of practical applications.

In order to reduce the feature dimension of EEG signals, some studies have shown that parametric feature extraction methods are successfully applied to EEG signal analyses [1012] and nonparametric feature extraction method proposed by one member of our research team is also proved the application on the recognition of satellite images [13]. Hence, after obtaining the EEG signals, refinement of analysis in this paper was first achieved by comparing data produced from three different feature extraction methods including nonparametric weighted feature extraction (NWFE), principal component analysis (PCA), linear discriminant analysis (LDA), which were applied to reduce the feature dimension and project the measured EEG signals to a feature space spanned by their eigenvectors. After that, the mapped data could be classified with fewer features and their classification results are compared by utilizing two different classifiers including $k$ nearest neighbor classification (KNNC) and naive bayes classifier (NBC). It should be noted that since we want to put emphasis on the efficacy comparison between the nonparametric and parametric feature extraction methods, we adopted two simple classifiers (KNNC and NBC) to avoid the good prediction results obtained mainly owing to adopting a complicated classifier even though Garrett et al. show that nonlinear classifiers produces slightly better prediction results for the classification of spontaneous EEG signals [14].

\section{SYSTEM ARCHITECTURE}

\subsection{Virtual reality-(VR-) based driving environment}

To explore brain activities in the safety-driving system, this experiment was designed to detect and analyze the event related potential (ERP) signals of brain activities related to traffic light events (Red-Green-Yellow) since they are the most frequent events when driving on the roads and highly correlated to the traffic accidents. The overall experiment environment included various simulated events shown in Figure 1: (1) a visual display unit to provide the simulated events, (2) EEG measurement system with 36-channel EEG

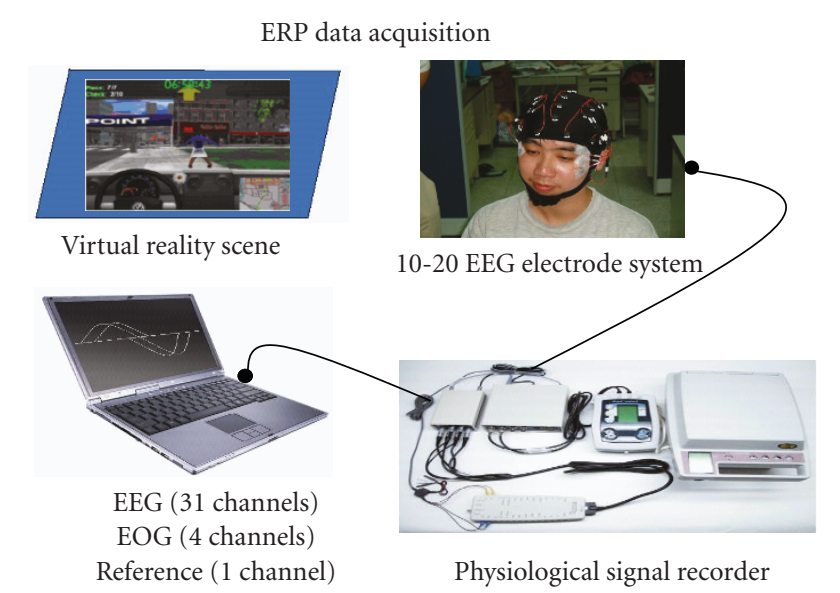

FIGURe 1: Physiological signal measurement system in the VR-based traffic-light simulation experiments.

head mounted sensors, and (3) spatial and temporal signal processing technologies based on several kinds of feature extraction and classification methods. Figure 2 shows the flowchart of EEG signal processing and analysis. EEG signals are digitally sampled and one among these channels is selected for classification task. Each dataset for one subject was shuffled and randomly divided into 4 subdatasets to do 4 -fold cross-validation. In each cross-validation stage it includes 4 runs. Each run will use 3 subdatasets for training and the remaining one subdataset for testing. Labeling the subdatasets from 1 to 4 , then the training data used in each run would be $\{2,3,4\},\{1,3,4\},\{1,2,4\}$, and $\{1,2,3\}$, respectively, while the test data are $\{1\},\{2\},\{3\}$, and $\{4\}$, accordingly. The average accuracy of the 4 runs is calculated as the result of this cross-validation stage. Such processes were repeated 10 times to get the average accuracy and standard deviation to decrease possible bias caused by any specific selected dataset. As shown in Figure 2, three different feature extraction methods including nonparametric weighted feature extraction (NWFE), principal component analysis (PCA), and linear discriminant analysis (LDA) are used. After feature extraction process, the extracted features are classified by two different classifiers: $k$ nearest neighbor classification (KNNC) and naive bayes classifier (NBC). The classification results of different combinations of features extraction approaches and classification methods are evaluated and compared.

\subsection{Subjects and EEG data collection}

Six subjects (ages from 20 to 40 years) participated in the VRbased traffic-light driving experiments, where EEG signals were simultaneously recorded. The subject was asked to decelerate/stop the car by pressing the right button of a joystick using the right hand when he/she detected a red light, to accelerate the car by pressing the left button using the left hand when he/she saw a yellow light, and do nothing (keep constant speed) when he/she saw a green light. Thirtysix EEG/EOG channels (using sintered Ag/AgCl electrodes 


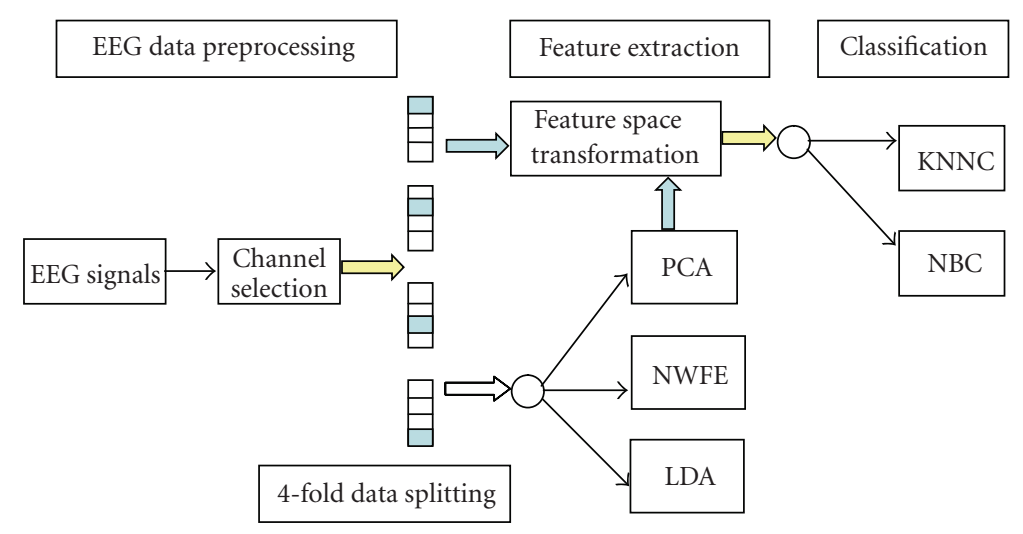

FIGURE 2: System flowchart for processing the EEG signals.

with a unipolar reference at the right earlobe), 2 ECG channels (bipolar connection) were simultaneously recorded by the Scan NuAmps Express system (Compumedics Ltd., VIC, Australia). All the EEG/EOG sensors were placed based on a modified international 10-20 system. Before data acquisition, the contact impedance between EEG electrodes and scalp was controlled to be less than $5 \mathrm{k} \Omega$. The EEG data were recorded with 16-bit quantization level at a sampling rate up to $1 \mathrm{KHz}$. Then, EEG data were preprocessed using a simple low-pass filter with a cutoff frequency of $50 \mathrm{~Hz}$ to remove the line noise $(60 \mathrm{~Hz}$ and its harmonic) and other high-frequency noise for further analysis.

\section{FEATURE EXTRACTION AND CLASSIFICATION METHODS}

To analyze the relation between EEG signals and the subjects' response, we apply the three different feature extraction and classification methods on different channel signals. According to several experimental test runs, we select $\mathrm{Pz}$ as the target channel for classification. Though some other channels may yield similar level of classification accuracy such as $\mathrm{Cz}$, however, our goal is focused on the sensory visual processing signal rather than others, so we make the decision to choose $\mathrm{Pz}$ channel. Figure 2 shows the system flowchart for processing the EEG signals. The Pz channel EEG signals are processed through one of feature extraction methods among nonparametric weighted feature extraction (NWFE), principal component analysis (PCA), or linear discriminant analysis (LDA) to map the original data to feature vectors of reduced dimension. After that, the reduced features are fed into one of classifiers such as $k$ nearest neighbor classifier (kNNC) and naive bayes classifier (NBC) for comparison of classification accuracy. Each analysis method is described briefly in this section.

\subsection{Nonparametric weighted feature extraction (NWFE)}

NWFE is a nonparametric feature extraction [13]. The main ideals of NWFE are putting different weights on every sample to compute the "weighted means" and compute the distance between samples and their weighted means as their "closeness" to boundary, then defining nonparametric between-class and within-class scatter matrices which put large weights on the samples close to the boundary and de-emphasize those samples far from the boundary. The between-class scatter matrix $S_{b}^{\mathrm{NW}}$ and the within-class scatter matrix $S_{w}^{\mathrm{NW}}$ of NWFE are defined as

$$
\begin{aligned}
& \left.S_{b}^{\mathrm{NW}}=\sum_{i=1}^{L} P_{i} \sum_{\substack{j=1 \\
j \neq i}}^{L} \sum_{\substack{N_{i} \\
N_{i}}}^{\lambda_{k}^{(i, j)}} \frac{n_{i}}{n_{i}(i)}-M_{j}\left(x_{k}^{(i)}\right)\right)\left(x_{k}^{(i)}-M_{j}\left(x_{k}^{(i)}\right)\right)^{T}, \\
& S_{w}^{\mathrm{NW}}=\sum_{i=1}^{L} P_{i} \sum_{k=1}^{N_{i}} \frac{\lambda_{k}^{(i, i)}}{n_{i}}\left(x_{k}^{(i)}-M_{i}\left(x_{k}^{(i)}\right)\right)\left(x_{k}^{(i)}-M_{i}\left(x_{k}^{(i)}\right)\right)^{T},
\end{aligned}
$$

where $M_{j}\left(x_{k}^{(i)}\right)=\sum_{l=1}^{N_{j}} w_{k l}^{(i, j)} x_{l}^{(j)}$,

$$
\begin{aligned}
\lambda_{k}^{(i, j)} & =\frac{\operatorname{dist}\left(x_{k}^{(i)}, M_{j}\left(x_{k}^{(i)}\right)\right)^{-1}}{\sum_{t=1}^{N_{j}} \operatorname{dist}\left(x_{t}^{(i)}, M_{j}\left(x_{t}^{(i)}\right)\right)^{-1}}, \\
w_{k l}^{(i, j)} & =\frac{\operatorname{dist}\left(x_{k}^{(i)}, x_{l}^{(j)}\right)^{-1}}{\sum_{t=1}^{n_{j}} \operatorname{dist}\left(x_{t}^{(i)}, x_{l}^{(j)}\right)^{-1}}
\end{aligned}
$$

where $N_{i}$ denotes the training sample size of class $i, L$ is the number of classes, $P_{i}$ is a prior probability of class $i, x_{l}^{(i)}$ is the $l$ th sample of class $i, M_{j}\left(x_{l}^{(i)}\right)$ denotes the weighted mean of $x_{l}^{(i)}$ in class $j$, and $\operatorname{dist}(x, z)$ is the Euclidean distance from $x$ to $z$. If the distance between $x_{k}^{(i)}$ and $M_{j}\left(x_{k}^{(i)}\right)$ is small, then its weight $\lambda_{k}^{(i, j)}$ will be close to 1 ; otherwise, $\lambda_{k}^{(i, j)}$ will be close to 0 . The sum of the $\lambda_{k}^{(i, j)}$ for class $i$ is 1 . The weight $w_{k l}^{(i, j)}$ for computing weighted means is a function of $x_{k}^{(i)}$ and $x_{l}^{(j)}$. If the distance between $x_{k}^{(i)}$ and $x_{l}^{(j)}$ is small then its weight $w_{k l}^{(i, j)}$ will be close to 1 ; otherwise, $w_{k l}^{(i, j)}$ will be close to 0 . The sum of the $w_{k l}^{(i, j)}$ for $M_{j}\left(x_{k}^{(i)}\right)$ is 1 .

There are two advantages of using the nonparametric scatter matrices. First, they are generally of full rank. This provides the ability to specify the number of extracted features desired and to reduce the effect of the singularity 
problem. This is in contrast to linear discriminant analysis (LDA), which usually only can extract $L-1$ (number of classes minus one) features. In a real situation, this may not be enough. Second, the nonparametric nature of scatter matrices reduces the effects of outliers and works well even for nonnormal datasets. NWFE provides greater weight to samples near the expected decision boundary. This tends to provide for increased classification accuracy.

\subsection{Principlal component analysis (PCA)}

The goal of PCA is to find a set of $p \leq d$ vectors in $R^{d}$ space containing the maximum amount of variance in the data. Suppose that the data has been centered in the original space and $v$ is an arbitrary normalized vector in $R^{d}$. The variance of the projections of the all pixels $x_{j}$ onto this normalized direction $v$ is

$$
\frac{1}{N} \sum_{j=1}^{N} \nu^{T} x_{j} x_{j}^{T} \nu=v^{T}\left(\frac{1}{N} \sum_{j=1}^{N} x_{j} x_{j}^{T}\right) \nu=v^{T} C \nu,
$$

where $C$ is the $d \times d$ covariance matrix and defined by

$$
C=\frac{1}{N} \sum_{j=1}^{N} x_{j} x_{j}^{T} .
$$

The first principal vector can be found by the following equation:

$$
\nu=\underset{v \in R^{d},\|\nu\|=1}{\arg \max } v^{T} C \nu .
$$

The solution of the above equation is the eigenvector $v$ of $C$ with respect to the largest eigenvalue. One can seek for the direction of second largest variance in the orthogonal subspace, by looking for the largest eigenvector in the matrix obtained by deflating the matrix $C$ with respect to $v$. Repeating this step shows that the mutually orthogonal directions of maximum variance in order of decreasing size are given by the eigenvectors of $C$.

\subsection{Linear discriminant analysis (LDA)}

LDA is often used for dimension reduction in classification problems. It is also called the parametric feature extraction method in [15], since LDA uses the mean vector and covariance matrix of each class. Usually within-class, between class, and mixture scatter matrices are used to formulate the criterion of class separability. A within-class scatter matrix for $L$ classes is expressed by

$$
S_{w}^{\mathrm{LDA}}=\sum_{i=1}^{L} P_{i} \Sigma_{i}=\sum_{i=1}^{L} P_{i} S_{w_{i}}^{\mathrm{LDA}}
$$

where $P_{i}$ denotes the prior probability of class $i$ and $\Sigma_{i}$ is the class covariance matrix. A between-class scatter matrix is expressed as

$$
\begin{aligned}
S_{b}^{\mathrm{LDA}} & =\sum P_{i}\left(m_{i}-m_{0}\right)\left(m_{i}-m_{0}\right)^{T} \\
& =\sum_{i=1}^{L-1} \sum_{j=i+1}^{L} P_{i} P_{j}\left(m_{i}-m_{j}\right)\left(m_{i}-m_{j}\right)^{T},
\end{aligned}
$$

where $m_{i}$ is the class mean and $m_{0}$ represents the expected vector of the mixture distribution and is given by

$$
m_{0}=\sum_{i=1}^{L} P_{i} m_{i}
$$

The optimal features are determined by optimizing the Fisher criterion given by

$$
J_{\mathrm{LDA}}=\operatorname{tr}\left[\left(S_{w}^{\mathrm{LDA}}\right)^{-1} S_{b}^{\mathrm{LDA}}\right]
$$

This is equivalent to solve the following generalized eigenvalue problem and the extracted eigenvectors are formed the transformation matrix of LDA,

$$
S_{b}^{\mathrm{LDA}} v=\lambda S_{w}^{\mathrm{LDA}} v
$$

\subsection{Classifiers}

In this study, two types of classifiers are used.

(i) $k$ nearest neighbor classification $(\mathrm{kNNC})$ is a simple and appealing approach in pattern recognition. $k$ nearest neighbor classifier finds the set of $k$ nearest neighbors in the training set to a testing sample, $x$, and then classifies $x$ as the most frequent class among the $k$ neighbors. The search of nearest neighbors is a flexible classification scheme, and does not involve any preprocessing or fitting of the training data. Hence, $k$ nearest neighbor classifier belongs to nonparametric classifiers. The detailed definition of nearest neighbor classification is described as in [15]. In this paper, the parameter $k$ is set to 1 or 3 and the classifiers are denoted as KNN1 or KNN3 in the following paragraphs.

(ii) Naive bayes classifier (NBC) is also a simple approach in pattern recognition. Based on Bayesian theorem and assumption of independence of attributes, NBC tries to appoint a sample $x$ with attribute values $\left(A_{1}=v_{1}, A_{2}=v_{2}, \ldots, A_{n}=v_{n}\right)$ to class $C_{i}$ with maximum a posterior probability (MAP) for all classes $i$,

$$
\begin{aligned}
c^{*} & =\arg \max _{i} \operatorname{Prob}\left(C_{i} \mid\left(\nu_{1}, v_{2}, \ldots, v_{n}\right)\right) \\
& =\arg \max _{i} \frac{P\left(\left(\nu_{1}, v_{2}, \ldots, v_{n}\right) \mid C_{i}\right) P\left(C_{i}\right)}{P\left(\nu_{1}, \nu_{2}, \ldots, v_{n}\right)} \\
& =\arg \max _{i} P\left(\left(\nu_{1}, \nu_{2}, \ldots, v_{n}\right) \mid C_{i}\right) P\left(C_{i}\right) \\
& =\arg \max _{i} \prod_{h=1}^{n} P\left(\nu_{h} \mid C_{i}\right) P\left(C_{i}\right),
\end{aligned}
$$

where $P\left(v_{h} \mid C_{i}\right)$ is the probability of the attribute $A_{h}=\nu_{h}$ given the class $C_{i}$ and $P\left(C_{i}\right)$ is the number of training samples belonging to class $C_{i}$. Due to the precise nature of the probability model, we can quickly finish the training process for NBC. And in many research topics NBC has shown its better performance than other kinds of classifiers. 


\subsection{Performance measure}

Two performance measures are used for the evaluation of classification result. One is commonly used average classification accuracy, which is defined as

$$
\overline{P_{a}}=\left(\overline{\frac{N_{\text {correct }}}{N_{\text {total }}}}\right),
$$

where $N_{\text {correct }}$ is the number of correctly predicted samples and $N_{\text {total }}$ is the number of all testing samples.

The other performance measure is Kappa coefficient, also known as Cohen's Kappa or kappa statistic [16]. The Kappa coefficient value is ranged between 1 and -1 , which corresponds to a perfect and a completely wrong classification, respectively. And Kappa coefficient with value 0 means random guess performance. Following the derivation of Kappa [17], it can be calculated as follows;

$$
K=\frac{\left(P_{o}-P_{e}\right)}{\left(1-P_{e}\right)},
$$

where $P_{o}$ denotes the proportion of overall agreement and $P_{e}$ is the proportion of chance expected agreement.

\section{EXPERIMENT RESULTS}

In our experiment, a subject simulated driving a car using the VR-based ERP experimental system described in Section 2. The continuous EEG signals measured from the EEG sensors were first separated into several epochs/trials. For this experiment, an epoch or a trial was defined as containing the sampled data from -200 millseconds to 1000 millisecondss when a light event was given at 0 millisecond. The objective of this experiment was to detect and evaluate cognitive responses of the driver to traffic-light events by directly accessing brain signals originating in one channel of the visual region of the brain. Each EEG trial originally containing 1200 recorded values taken at intervals of 1200 milliseconds was downsampled by picking up the first sample of every 3 data points ( 3 milliseconds) to form one 400-dimension sample vector. The down-sampled EEG trials of every participant form the dataset for training and testing and each trial is classified into one of the red, green, or yellow light events (i.e., the number of class labels $L=3$ ). Each dataset for one subject was shuffled and randomly divided into 4 subdatasets to do 4 -fold cross-validation. Such processes were repeated 10 times to get the average accuracy and standard deviation to decrease possible bias caused by any specific selected dataset. Moreover, in order to increase computational efficiency, we applied three different feature extraction techniques to the dataset, including NWFE, PCA, and LDA. After feature extraction, the 400-dimension sample vectors for each participant were mapped to the feature space and the top one to fifty transformed features were chosen for testing optimal classification accuracy by observing the Kappa coefficients.

In performing the experiments, first, we evaluated how different EEG channels influence the classification accuracy. In order to find the channel most related to visual cognitive

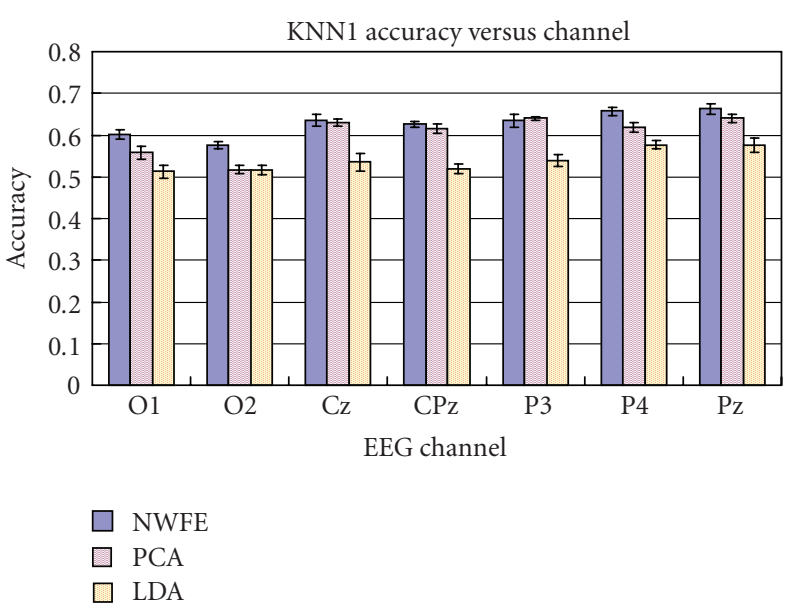

(a)
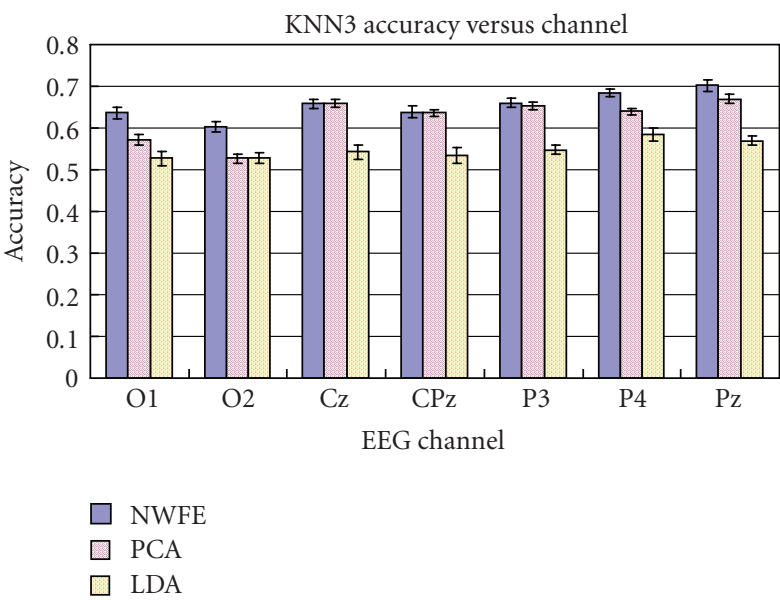

(b)

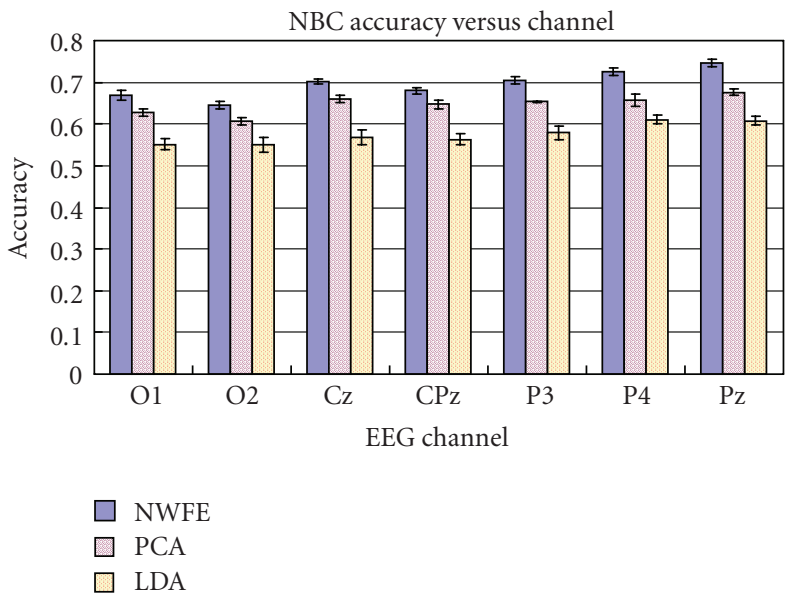

(c)

Figure 3: Accuracy plot for different EEG channels.

response, channels such as $\mathrm{O} 1, \mathrm{O} 2, \mathrm{Cz}, \mathrm{CPz}, \mathrm{P} 3, \mathrm{P} 4$, and $\mathrm{Pz}$ were tested. The average accuracy and standard deviation using KNN1, KNN3, and NBC classifiers were plotted in Figure 3 using the optimal feature number most suitable for each channel. From the data in Figure 3 for the KNN1 classifier, it can be seen that data from the $\mathrm{Pz}$ channel 


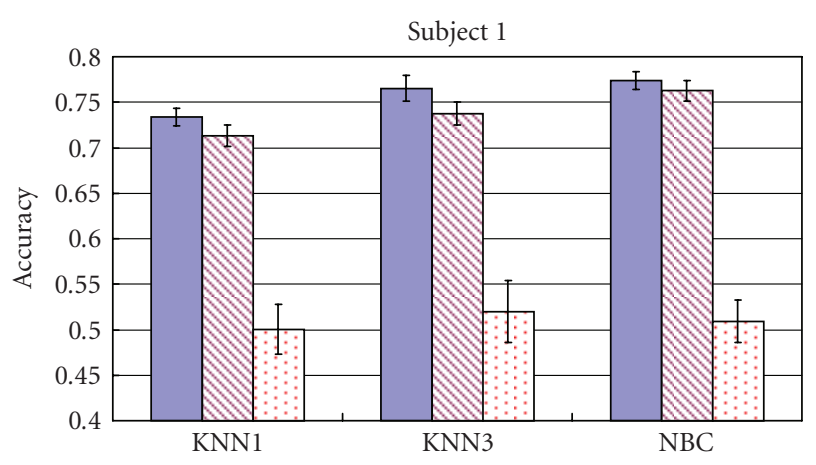

(a)

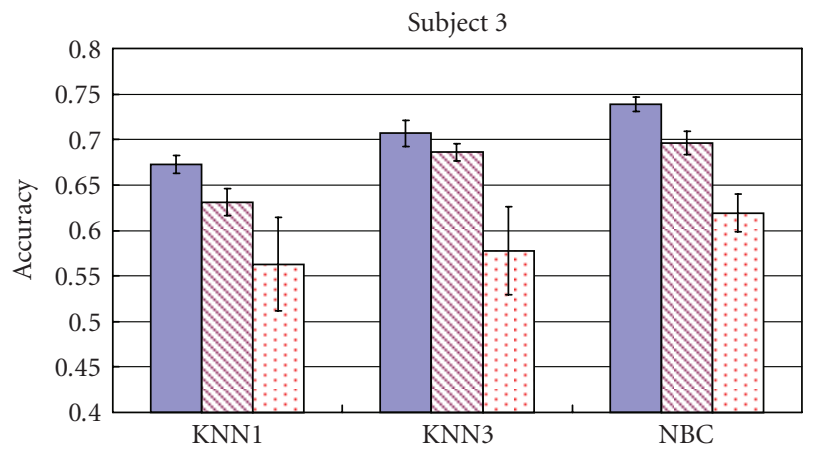

(c)

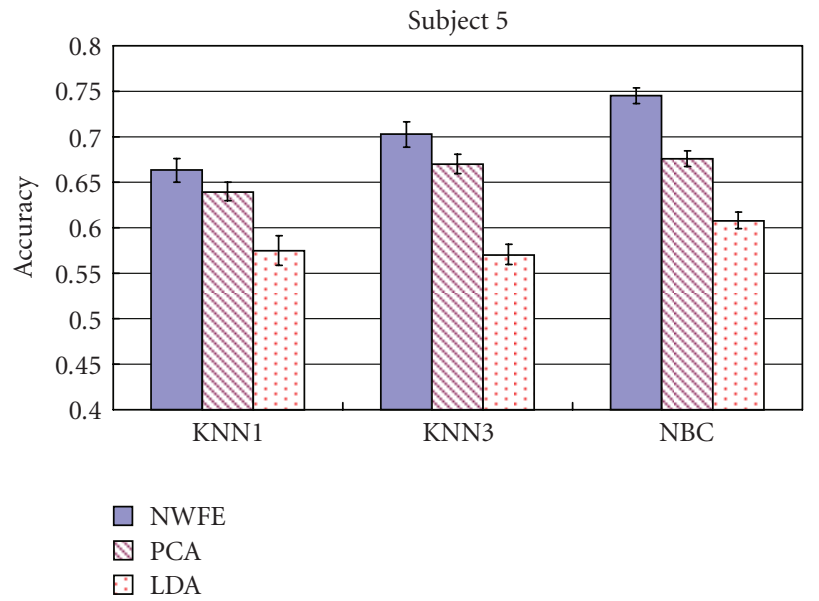

(e)

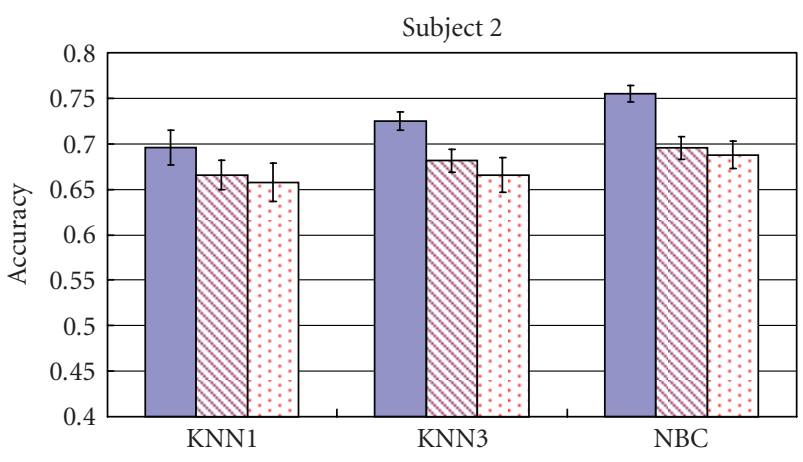

(b)

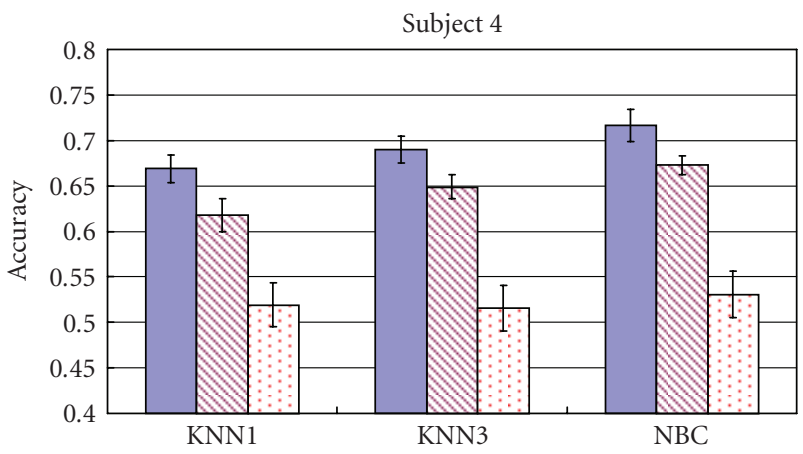

(d)

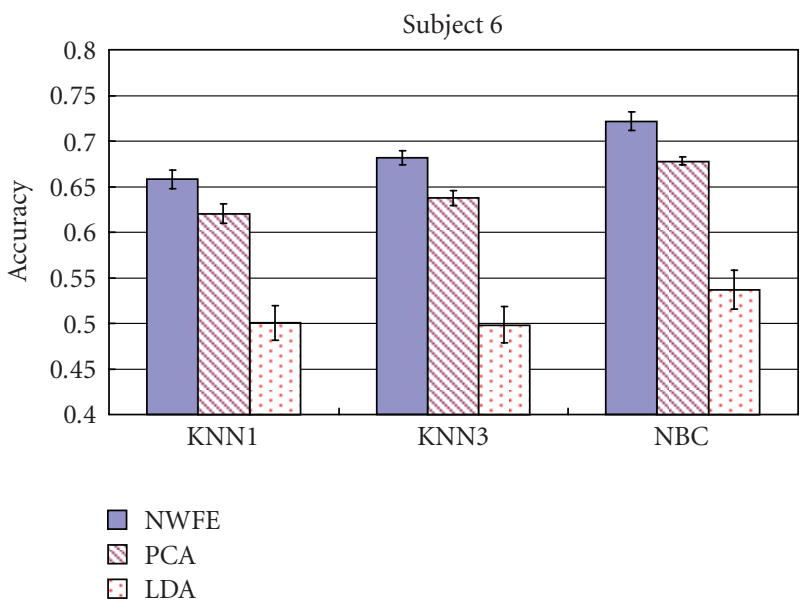

(f)

FIgURE 4: Classification results for six different subjects based on EEG signals from Pz channel.

performed best overall in more accurately predicting the cognitive response. For the NWFE and PCA feature extraction methods, Figure 3 shows that the $\mathrm{Pz}$ channel consistently performed best. Only for the LDA feature extraction method did the P4 channel perform better, but such performance was only marginally better. The same can be observed for the KNN3 and NBC classifiers (with the exception for the $\mathrm{Cz}$ channel for the KNN3 method, which performed on a par with the Pz channel). Consequently, in the subsequent test cases, we chose the measured signals of the $\mathrm{Pz}$ channel to be the feature vectors for classification.
Figure 4 shows the classification results of the 6 subjects using the different feature extraction methods and different classifiers. The chart shows that the choice of feature extraction methods and classifiers makes a significant difference. For instance, the NWFE feature extraction method combined with any other classifier produced consistently higher accuracy. Moreover, as shown in Figure 4, the NWFE+ NBC give the best classification accuracy for these subjects, and in fact the improvement in classification accuracy is $10 \% \sim 24 \%$ higher than LDA + KNN1. Also for all of the subjects, the LDA feature extraction method combined with 
any one of the classifiers produced the lowest accuracy, and in some instances, substantially poorer accuracy. It is possible that LDA itself can act as a classifier (e.g., use Fisher's classification functions) and its performance as a classifier might be better than using the LD scores into a different classifier. The PCA feature extraction method, combined with any of the classifiers, produced results consistently better than the LDA method, but less accurate (and in some cases significantly less accurate) than the NWFE method. Table 1 contains amplification of the data from Figure 4, supplying in addition the standard deviation for accuracy for each subject by each method,and also the numbers of features used. Also as can be seen in Table 1, the NWFE method effectively reduces the number of features from 400 down to 2. Since the amplitudes of single-trial ERP signals are far weaker than the ongoing EEG signals, proper feature extraction before classification can increase the accuracy and reduce the data dimension to save the training and processing time for online analysis system.

In order to confirm the efficacy of the NWFE feature extraction method, when used with any classifier, the same data was analyzed using an alternative index, Kappa coefficient. Figure 5 shows the Kappa coefficient versus a range of features for Subject 3 using different classifiers. The $x$-axis ranging from 1 to 50 represents the number of features used for classification after feature reduction, and the $y$-axis represents the Kappa coefficient. A higher Kappa coefficient indicates higher accuracy. Figure 5 offers further corroboration that NWFE obtains better accuracy than other methods, especially when using fewer features. It is noted that as feature numbers increase, initially the accuracy also increases, but after best accuracy achieved by using a certain number of features, the accuracy begins to decrease when more features are used. Also,different classifiers differ somewhat in how the accuracy decreases [18].

In the previous VR-based stop light experiment without the dynamic platform [7], the recognition results between two lights (red/yellow) were $67 \%$ to $73 \%$ in average of five subjects with different algorithms. In our past study, we acquired 31-channel whole head EEG signals to recognize traffic-light visual ERP [9]. The recognition sensitivity can achieve $79 \%$ to $95 \%$ by combining independent component analysis (ICA), PCA, and self-constructing neural fuzzy inference network (SONFIN). In this study, we utilize NWFE to extract features from one channel single-trial EEG data and use NBC for classification. It can achieve up to $77 \%$ accuracy in traffic-light visual ERP classification. Since only one EEG channel is used, the preparation time for electrode placement, skin preparation, and gel application as well as the system complexity can be greatly reduced. It is more convenient for practical applications. On the other hand, the accuracy is possibly improved by some other choices of classifiers. For instance, we have some preliminary investigation by using the support vector machine (SVM) with RBF kernel to classify the ERPs of Subject 3 as an example. The accuracy of applying SVM to classify the features extracted by NWFE, PCA, and LDA is $74.87 \%$, $72.86 \%$, and $59.6 \%$, respectively. The NWFE + SVM can also

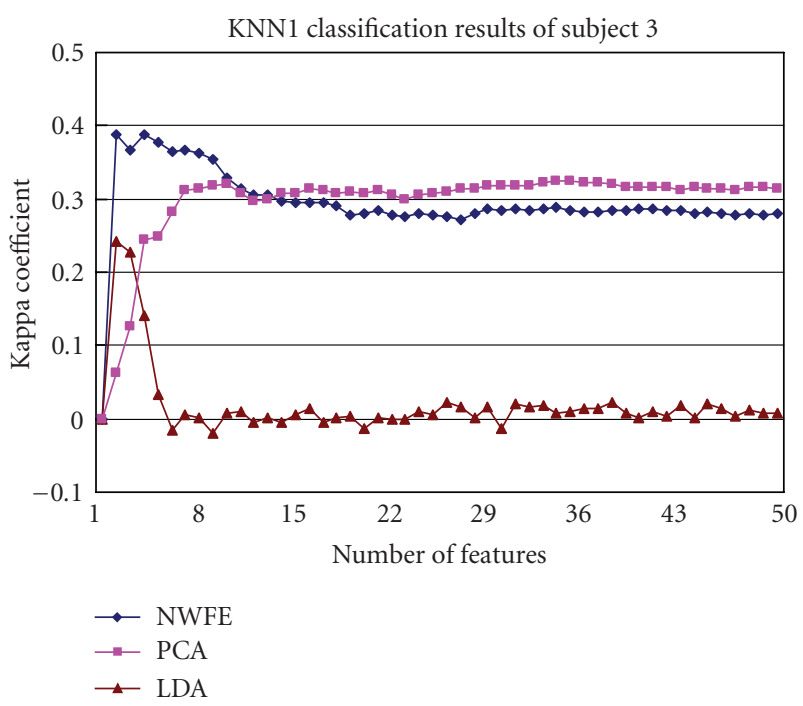

(a)

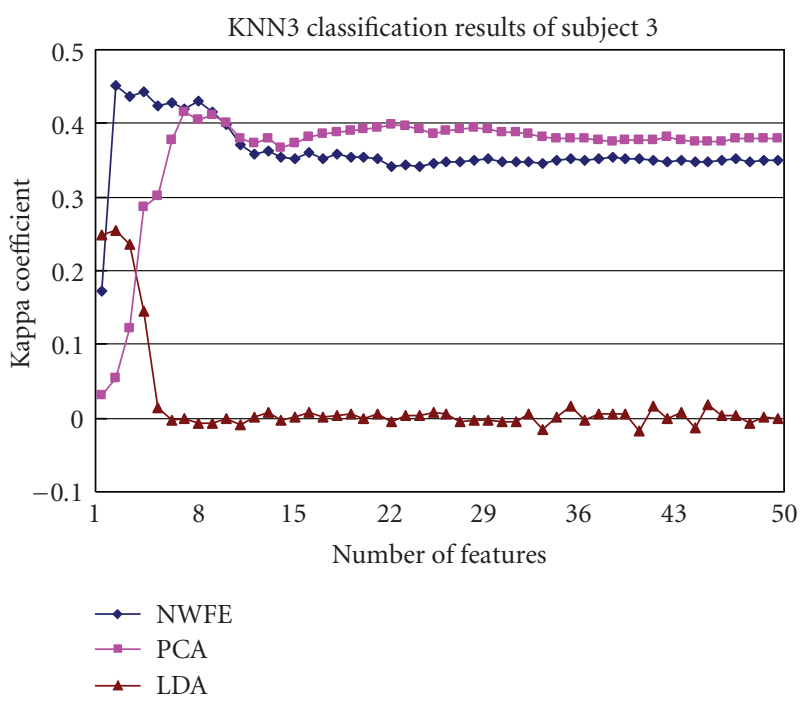

(b)

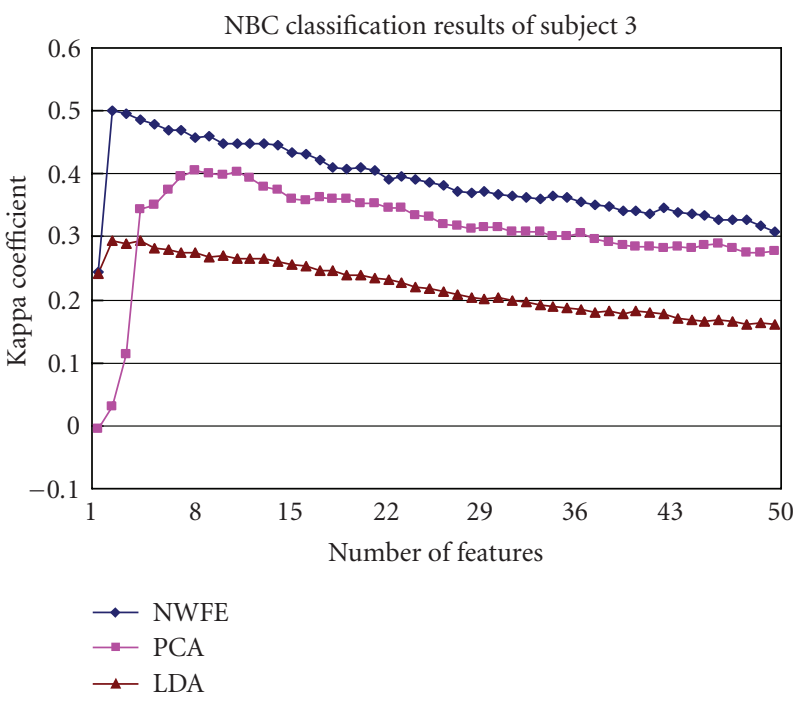

(c)

Figure 5: Kappa coefficient versus the number of features (subject $3)$. 

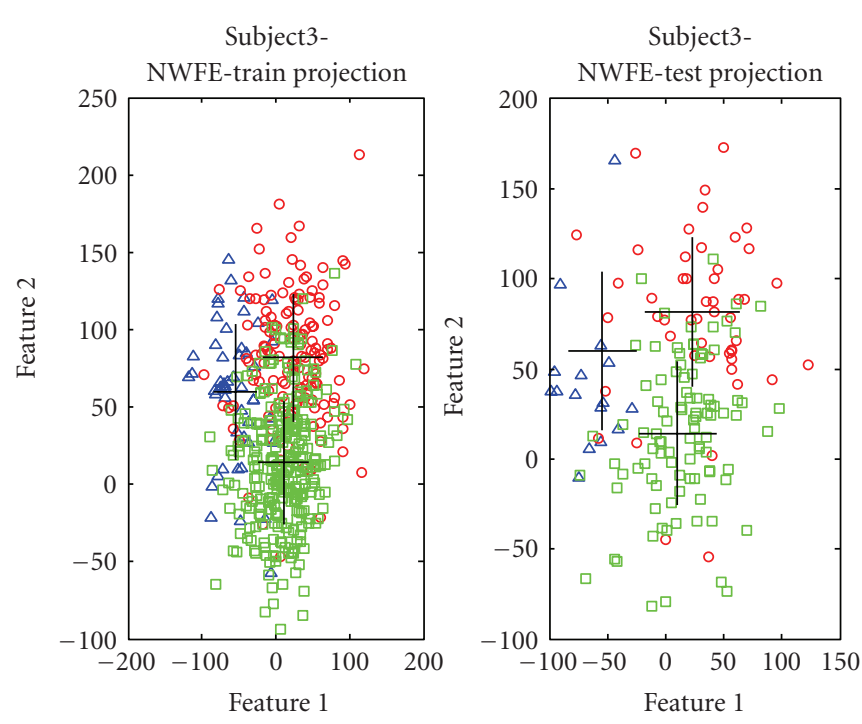

(a) NWFE-mapped feature space
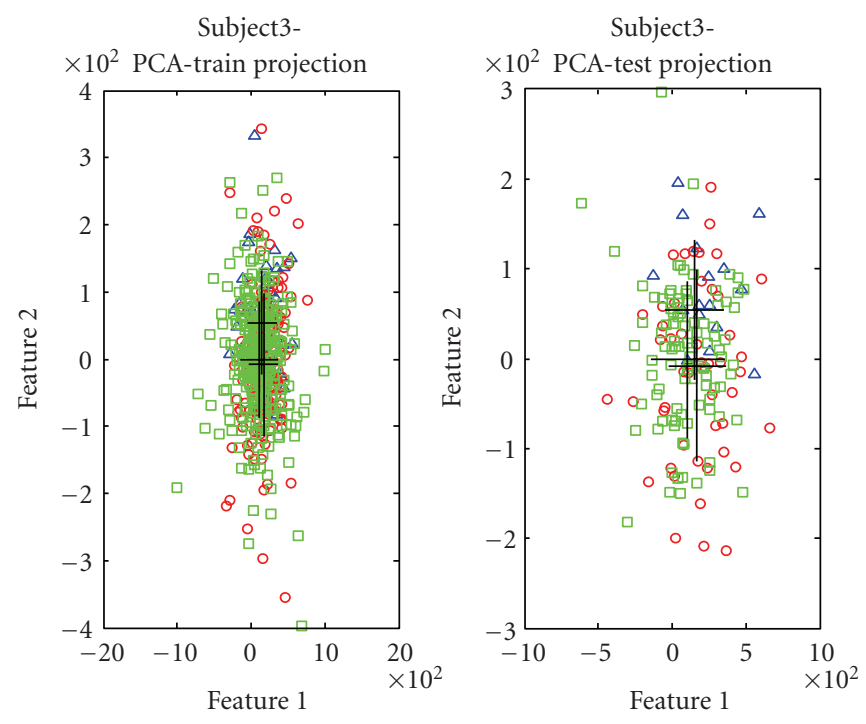

(b) PCA-mapped feature space

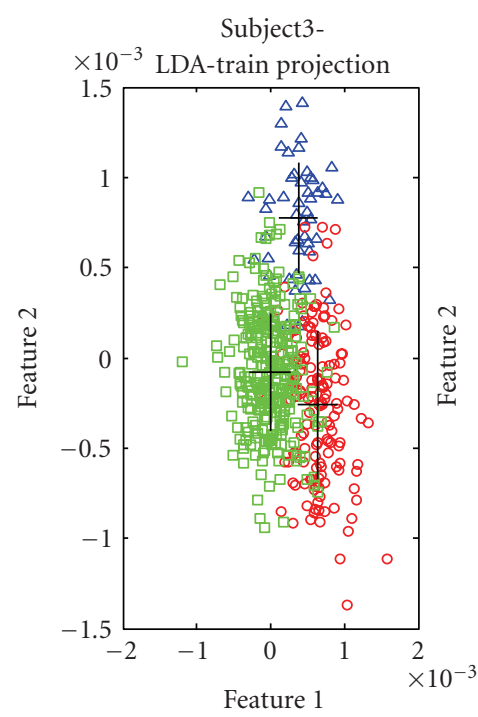

Subject3-

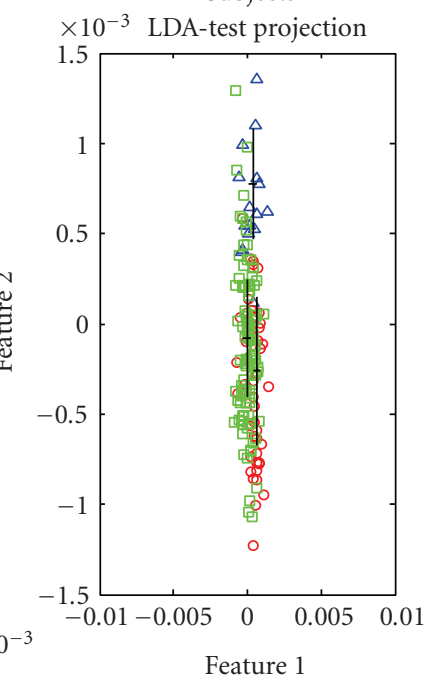

(c) LDA-mapped feature space

Figure 6: Scatter-plot for Subject 3 using (a) NWFE, (b) PCA, and (c) LDA methods.

achieve the best performance and is slightly better than the NWFE + NBC in this case.

To graphically visualize the difference of projected data spread in a reduced feature space, training, and testing data are projected into a 2 -dimension feature space. Three scatter plots are shown in Figure 6(a) through Figure 6(c), where the left-hand side is the training data projection and the right-hand side is the test data projection. Three colors are used to indicate the three different EEG events (e.g., red, green, and yellow lights) and each focus indicates the center of each event distribution. From the Figures, NWFE and LDA both separate projected training data better than PCA, while NWFE also performs better than LDA for projected test data separation. The distinct separation of the three event signals for both training and testing patterns shows the overall superiority of the NWFE method. That gives a visual interpretation for the classification results.

\section{CONCLUSIONS}

In this study, we develop a simple and feasible algorithm for single-trial classification of traffic-light stimuli experiments. Using single channel to acquire EEG signal and utilizing the fewer features are the advantages of the proposed algorithm in practical applications. We present quantitative and comparative techniques for ongoing assessment of drivers' cognitive responses by analysis of EEG signals in $3 \mathrm{D}$ virtual-reality-based dynamic driving environment. This 
TABLE 1: Classification results.

\begin{tabular}{|c|c|c|c|c|c|c|c|}
\hline \multicolumn{8}{|c|}{ CL: classifier, Acc: accuracy, Fno: feature no. used } \\
\hline \multicolumn{2}{|c|}{ Feature extraction } & \multicolumn{2}{|l|}{ NWFE } & \multicolumn{2}{|l|}{ PCA } & \multicolumn{2}{|l|}{ LDA } \\
\hline Subject & $\mathrm{CL}$ & Acc & Fno & Acc & Fno & Acc & Fno \\
\hline \multirow{3}{*}{ Sub 1} & KNN1 & $73.33 \% \pm 0.97 \%$ & 2 & $71.29 \% \pm 1.18 \%$ & 11 & $50.01 \% \pm 2.73 \%$ & 2 \\
\hline & KNN3 & $76.51 \% \pm 1.43 \%$ & 3 & $73.78 \% \pm 1.28 \%$ & 11 & $52.04 \% \pm 3.42 \%$ & 3 \\
\hline & NBC & $77.37 \% \pm 0.94 \%$ & 2 & $76.26 \% \pm 1.15 \%$ & 3 & $50.95 \% \pm 2.33 \%$ & 8 \\
\hline \multirow{3}{*}{ Sub 2} & KNN1 & $69.57 \% \pm 1.89 \%$ & 3 & $66.56 \% \pm 1.62 \%$ & 7 & $65.74 \% \pm 2.10 \%$ & 2 \\
\hline & KNN3 & $72.50 \% \pm 1.03 \%$ & 2 & $68.13 \% \pm 1.22 \%$ & 32 & $66.60 \% \pm 1.91 \%$ & 2 \\
\hline & NBC & $75.50 \% \pm 0.93 \%$ & 2 & $69.54 \% \pm 1.26 \%$ & 11 & $68.77 \% \pm 1.49 \%$ & 1 \\
\hline \multirow{3}{*}{ Sub 3} & KNN1 & $67.22 \% \pm 0.98 \%$ & 4 & $63.12 \% \pm 1.49 \%$ & 35 & $56.30 \% \pm 5.16 \%$ & 2 \\
\hline & KNN3 & $70.69 \% \pm 1.43 \%$ & 2 & $68.61 \% \pm 0.95 \%$ & 7 & $57.81 \% \pm 4.83 \%$ & 2 \\
\hline & NBC & $73.84 \% \pm 0.80 \%$ & 2 & $69.63 \% \pm 1.28 \%$ & 8 & $61.92 \% \pm 2.06 \%$ & 4 \\
\hline \multirow{3}{*}{ Sub 4} & KNN1 & $66.88 \% \pm 1.50 \%$ & 3 & $61.77 \% \pm 1.77 \%$ & 17 & $51.91 \% \pm 2.42 \%$ & 1 \\
\hline & KNN3 & $69.02 \% \pm 1.49 \%$ & 3 & $64.91 \% \pm 1.35 \%$ & 20 & $51.57 \% \pm 2.48 \%$ & 1 \\
\hline & NBC & $71.66 \% \pm 1.75 \%$ & 6 & $67.28 \% \pm 1.00 \%$ & 4 & $53.05 \% \pm 2.58 \%$ & 1 \\
\hline \multirow{3}{*}{ Sub 5} & KNN1 & $66.30 \% \pm 1.27 \%$ & 2 & $63.98 \% \pm 1.04 \%$ & 12 & $57.52 \% \pm 1.64 \%$ & 3 \\
\hline & KNN3 & $70.25 \% \pm 1.39 \%$ & 2 & $67.03 \% \pm 1.06 \%$ & 22 & $57.03 \% \pm 1.11 \%$ & 2 \\
\hline & NBC & $74.53 \% \pm 0.90 \%$ & 2 & $67.60 \% \pm 0.83 \%$ & 13 & $60.79 \% \pm 0.93 \%$ & 1 \\
\hline \multirow{3}{*}{ Sub 6} & KNN1 & $64.92 \% \pm 1.71 \%$ & 3 & $62.32 \% \pm 0.93 \%$ & 13 & $53.89 \% \pm 1.90 \%$ & 1 \\
\hline & KNN3 & $67.41 \% \pm 0.79 \%$ & 2 & $65.73 \% \pm 0.55 \%$ & 12 & $54.80 \% \pm 0.71 \%$ & 3 \\
\hline & NBC & $71.40 \% \pm 0.63 \%$ & 2 & $68.21 \% \pm 0.82 \%$ & 7 & $57.14 \% \pm 1.91 \%$ & 1 \\
\hline
\end{tabular}

environment consists of surrounded virtual reality scenes, a driving motion simulator, and the EEG signal acquisition system. Regarding comparison of classification results, we combined three well-known feature extraction methods with two different classifiers to classify the single-trial EEG signal of driver's cognitive responses corresponding to different traffic-light events. Experimental results showed that NWFE could extract the fewer features (almost in 2 features) in each subject experiment to represent the EEG data distribution and then using NBC as the classifier could reach the better accuracy. In addition, since the proposed approach utilizes only one EEG channel, the preparation time for electrode placement, skin preparation, and gel application as well as the system complexity can be greatly reduced. It is feasible and desirable for practical applications.

\section{ACKNOWLEDGMENTS}

This work was supported in part by the National Science Council, Taiwan, under Contracts NSC 96-2627-E-009-001and NSC 96-2218-E-009-001-, and in part by the "Aiming for the Top University Plan (ATU)" of the National Chiao-Tung University and the Ministry of Education (MOE), Taiwan, under Contract no. 97W806.

\section{REFERENCES}

[1] A. Kemeny and F. Panerai, "Evaluating perception in driving simulation experiments," Trends in Cognitive Sciences, vol. 7, no. 1, pp. 31-37, 2003.

[2] Z. Duric, W. D. Gray, R. Heishman, et al., "Integrating perceptual and cognitive modeling for adaptive and intelligent human-computer interaction," Proceedings of the IEEE, vol. 90, no. 7, pp. 1272-1289, 2002.

[3] D. D. Schmorrow and A. A. Kruse, "DARPA's augmented cognition program-tomorrow's human computer interaction from vision to reality: building cognitively aware computational systems," in Proceedings of the 7th IEEE Conference on Human Factors and Power Plants, pp. 7/1-7/4, Scottsdale, Ariz, USA, September 2002.

[4] P. Sykacek, S. J. Roberts, and M. Stokes, "Adaptive BCI based on variational Bayesian Kalman filtering: an empirical evaluation," IEEE Transactions on Biomedical Engineering, vol. 51, no. 5, pp. 719-727, 2004.

[5] T. J. Dasey and E. Micheli-Tzanakou, "Detection of multiple sclerosis with visual evoked potentials-an unsupervised computational intelligence system," IEEE Transactions on Information Technology in Biomedicine, vol. 4, no. 3, pp. 216224, 2000.

[6] X. Gao, D. Xu, M. Cheng, and S. Gao, "A BCI-based environmental controller for the motion-disabled," IEEE Transactions on Neural Systems and Rehabilitation Engineering, vol. 11, no. 2, pp. 137-140, 2003.

[7] J. D. Bayliss and D. H. Ballard, "Single trial P3 epoch recognition in a virtual environment," Neurocomputing, vol. 32-33, pp. 637-642, 2000.

[8] J. D. Bayliss and D. H. Ballard, "Recognizing evoked potentials in a virtual environment," in Advances in Neural Information Processing Systems 12, pp. 3-9, MIT Press, Cambridge, Mass, USA, 2000.

[9] C.-T. Lin, I.-F. Chung, L.-W. Ko, Y.-C. Chen, S.-F. Liang, and J.-R. Duann, "EEG-Based assessment of driver cognitive responses in a dynamic virtual-reality driving environment," IEEE Transactions on Biomedical Engineering, vol. 54, no. 7, pp. 1349-1352, 2007.

[10] S. Cerutti, G. Chiarenza, D. Liberati, P. Mascellani, and G. Pavesi, "A parametric method of identification of single-trial 
event-related potentials in the brain," IEEE Transactions on Biomedical Engineering, vol. 35, no. 9, pp. 701-711, 1988.

[11] J. Pardy, S. Roberts, and L. Tarassenko, "A review of parametric modeling techniques for EEG analysis," Medical Engineering \& Physics, vol. 18, no. 1, pp. 2-11, 1996.

[12] D. P. Burke, S. P. Kelly, P. de Chazal, R. B. Reilly, and C. Finucane, "A parametric feature extraction and classification strategy for brain-computer interfacing," IEEE Transactions on Neural Systems and Rehabilitation Engineering, vol. 13, no. 1, pp. 12-17, 2005.

[13] B.-C. Kuo and D. A. Landgrebe, "Nonparametric weighted feature extraction for classification," IEEE Transactions on Geoscience and Remote Sensing, vol. 42, no. 5, pp. 1096-1105, 2004.

[14] D. Garrett, D. A. Peterson, C. W. Anderson, and M. H. Thaut, "Comparison of linear, nonlinear, feature selection methods for EEG signal classification," IEEE Transactions on Neural Systems and Rehabilitation Engineering, vol. 11, no. 2, pp. 141144, 2003.

[15] K. Fukunaga, Introduction to Statistical Pattern Recognition, chapters 9-10, Academic Press, San Diego, Calif, USA, 1990.

[16] H. C. Kraemer, Encyclopedia of Statistical Science, John Wiley \& Sons, New York, NY, USA, 1982.

[17] G. Townsend, B. Graimann, and G. Pfurtscheller, "A comparison of common spatial patterns with complex band power features in a four-class BCI experiment," IEEE Transactions on Biomedical Engineering, vol. 53, no. 4, pp. 642-651, 2006.

[18] T. Howley, M. G. Madden, M.-L. O'Connell, and A. G. Ryder, "The effect of principal component analysis on machine learning accuracy with high-dimensional spectral data," KnowledgeBased Systems, vol. 19, no. 5, pp. 363-370, 2006. 